\title{
Working with Raoul Bott: From Geometry to Physics
}

\author{
Michael Atiyah
}

\section{Introduction}

I was a close friend and collaborator of Raoul and our association lasted for 50 years from our first meeting at the Institute for Advanced Study in Princeton in 1955 to our last conference at Santa Barbara in 2005, shortly before his death. We wrote 12 joint papers together, published over the 20 years between $1964-1984$, but this represents only the visible part of our joint collaboration. Our mathematical interaction started soon after we first met and continued wherever and whenever we met.

We spent lengthy periods together at Princeton, Harvard, Cambridge, Oxford, Bonn while we also shared shorter conferences in India, China, Hungary, Italy,...

Raoul and I were both geometers in the broad sense, with interests spanning topology, differential and algebraic geometry and eventually theoretical physics. We had different backgrounds but over time we learnt from each other and our interests fused. What was important was that we shared a common view in which global topological ideas provided a general framework to understand complex problems. Analysis was often at the deep core but the beauty lay in the way in which global results emerged in an elegant fashion, exemplified in the 1950 s by the dominant role of sheaf cohomology in the hands of Cartan and Serre.

About half-way through our 50 years Raoul and I, together with Is Singer and a few others, were suddenly confronted with new ideas in the theoretical physics of gauge theories. The wide gap between mathematics and physics of the time suddenly acquired a new connecting link, a bridge over which ideas and techniques flowed in both directions. Over the next few years this developed into a flood with spectacular results. Our teacher in all this was Edward Witten whom we encountered in the mid-seventies when he was a Junior Fellow at Harvard. Young enough to absorb new mathematical ideas, but old enough to have all physics at his fingertips, he was the ideal tutor for the two of us, together with our collaborators and students. A whole new generation has now grown up, in which physicists can manipulate spectral sequences and mathematicians can talk glibly about quantization.

2000 Mathematics Subject Classification. Primary 01A60; Secondary 58-03.

This is the final form of the paper.

(C)2010 American Mathematical Society 
In retrospect I feel we are all fortunate to have been there at the right time - a great period in the history of mathematics and physics.

I have said many times in the past that I always felt that I was following in the footsteps of Hermann Weyl, that I was traversing territory that Hermann Weyl had previously explored. Certainly, as the pioneer of gauge theory, he would have been delighted with recent developments. I know that Raoul also felt he was a disciple of Weyl. It was indeed Weyl who plucked Raoul from obscurity at Carnegie Tech. and brought him to Princeton; and Lie groups, which lay at the heart of much of Weyl's work, were also central for Raoul.

I have written elsewhere [2] a biographical memoir of Raoul, in which I describe both his personality and his work, but our friendship is beautifully encapsulated by Raoul in the inscription he wrote for me in Volume 1 of his collected works (January 1995):

To Michael - comrade in arms and so many exploits, both in the wonderful world of mathematics and in the less perfect corporeal one, with admiration, gratitude and deep affection.

In surveying the corpus of Raoul's work I would like to pick out five themes which are at the heart of his mathematics. These are

- Morse Theory

- Lie Groups

- Loop Spaces

- Equivariant Cohomology

- Explicit Formulae

The role of the first four will become clear in the detailed descriptions that are in subsequent sections, but I should say something about the last theme, which is on a rather different footing. It refers not to content but to style.

For Raoul mathematics was a balance between general structure, which gave shape and cohesion, and fine detail, where the beauty lay in precision and elegance. Like architecture, mathematics has beauty at both large and small scales. For Raoul a beautiful explicit formula was the ultimate objective. It showed that you really understood and could tame the general theory. This attention to minute detail was a source of "creative tension" between us. Frequently I thought explicit formulae an unnecessary distraction, but Raoul's preference often paid off and I was partially converted. An explicit formula, as for example in a representative differential form for a cohomology class, can be informative and ultimately useful, as in Chern-Simons theory.

In the subsequent sections I have selected a number of the papers I wrote with Raoul which represent our best joint work and which illustrate the five themes above. While they deal with separate issues they are not totally independent and there are links between many of them.

\section{Bott periodicity and $K$-theory}

There is little doubt that the periodicity theorems for the homotopy of the classical groups [13] are Raoul's greatest discovery. They are beautifully simple, have

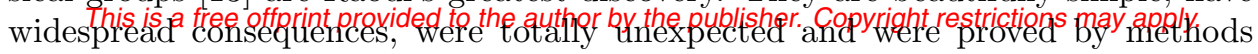
from analysis (Morse theory). 


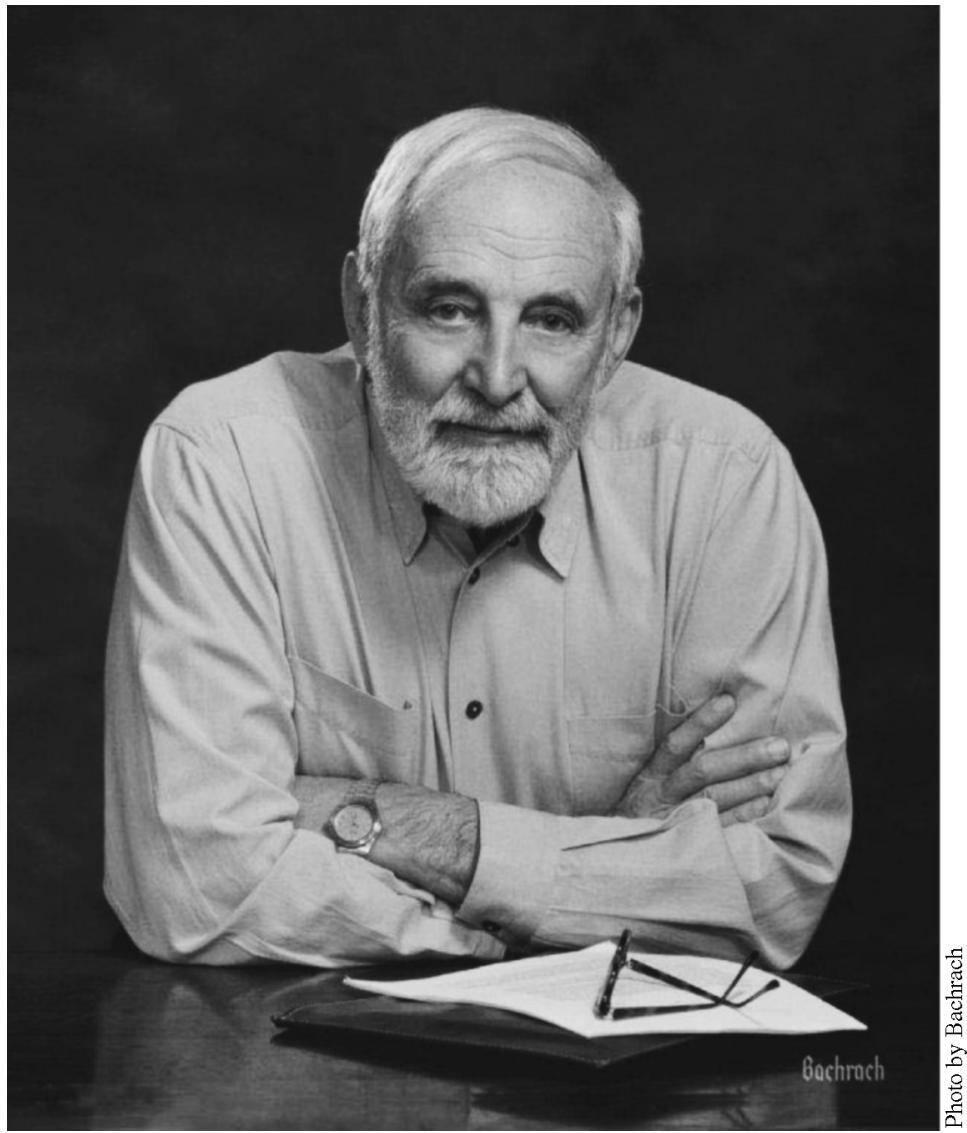

Figure 1. Raoul Bott

Let me first recall the Bott periodicity theorem for the unitary groups. Let

$$
\mathrm{U}=\lim _{N \rightarrow \infty} \mathrm{U}(N)
$$

be the stable unitary group, the limit being taken with respect to the standard inclusions $\mathrm{U}(N) \rightarrow \mathrm{U}(N+1)$. Then the Bott periodicity theorem asserts that there is a homotopy equivalence

$$
\Omega^{2} \mathrm{U} \sim \mathrm{U}
$$

where $\Omega^{2}$ denotes the double loop space. In particular the homotopy groups of $U$ are periodic with period 2 :

$$
\Pi_{q}(\mathrm{U})= \begin{cases}\mathbb{Z} & q \text { odd } \\ 0 & q \text { even }\end{cases}
$$

There is a similar periodicity for the stable orthogonal group $\mathrm{O}$, but here the period is 8 .

Bott's original proof came from Morse theory and was an outcome of his work

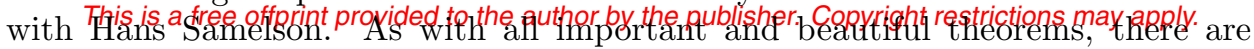
many different proofs of Bott periodicity, each reflecting some particular aspect and 
employing a variety of techniques. It is said that Gauss had eight proofs of quadratic reciprocity and there may be a similar number of proofs of Bott periodicity.

Bott periodicity takes an elegant form in the framework of $K$-theory as developed in topology by Hirzebruch and myself, following Grothendieck's revolutionary work in algebraic geometry. In $K$-theory, for any compact space $X$, the tensor product gives a natural homomorphism

$$
K(X) \otimes K\left(S^{2}\right) \rightarrow K\left(X \times S^{2}\right)
$$

and Bott periodicity essentially asserts that this is an isomorphism.

The history of this version is actually quite interesting. When Hirzebruch and I were developing the foundations of $K$-theory we stumbled across (1) and, in analogy with algebraic geometry, we automatically assumed this must be the Bott isomorphism. When preparing a Bourbaki seminar on $K$-theory I suddenly realized there was a gap at this point. I filled this by a roundabout route employing cohomology but felt this was unsatisfactory, especially as it would fail for the orthogonal group. In desperation I wrote to Raoul appealing for help. By looking carefully at his proof he was indeed able to show that it implied the isomorphism (1), and the gap was filled. This was all explained in [14], the only paper of Bott's that appeared in French, not a language that he was comfortable with. Later he regretted not knowing his translator so that he could be properly thanked!

Entirely different proofs of the periodicity theorem emerged many years later from the index theorem for elliptic operators, as I will describe in the next section.

\section{Index theory}

In algebraic geometry Grothendieck had introduced $K$-theory in order to give a new proof of the Hirzebruch - Riemann - Roch theorem, and to generalize it. When we move away from algebraic geometry to topology and differential equations, $K$ theory is again fundamental and plays a key role in the index theory of elliptic differential operators on manifolds.

The main theorem, developed by Singer and myself in the early sixties [12] gives an explicit formula for

$$
\text { index } D=\operatorname{dim}(\operatorname{ker} D)-\operatorname{dim}(\operatorname{coker} D)
$$

of an elliptic differential operator $D$ on a compact manifold $X$. The answer is given by an integral over $X$ of a differential form (or cohomology class) constructed from the symbol $\sigma_{D}$ of $D$. This depends only on the highest-order terms of $D$ and gives a map from the cotangent sphere bundle of $X$ into $\operatorname{GL}(N, \mathbb{C})$. This is for $D$ operating on $\mathbb{C}^{N}$-valued functions and there is a natural generalization when $D$ acts on sections of vector bundles.

In one direction the index theorem generalizes to families of elliptic operators $D_{y}$ parametrized by $y \in Y$. The index is then an element of $K(Y)$. This is close to Grothendieck's generalization and can be used to establish the periodicity theorem in the form (1) [1].

However there is another generalization of the index theorem which leads to an entirely different proof of (1). This involves the index theorem for manifolds $X$ with boundary $Z$. Here one needs a good notion of a boundary condition so that the index is still well defined. Such a notion had been identified earlier by analysts

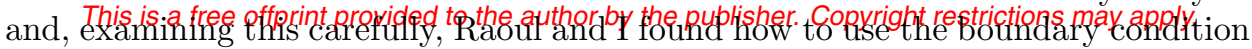
to extend the symbol from the cotangent sphere bundle $S(X)$ to its interior over $Z$. 


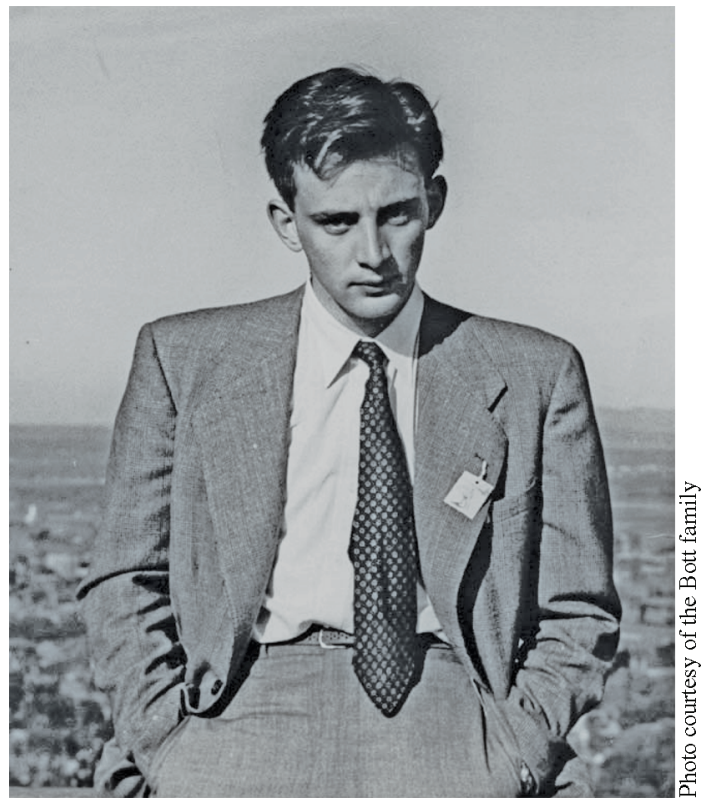

Figure 2. Raoul Bott during his McGill years at Parc du MontRoyal, 1942

This meant that the integral formula for the index of $D$ could now be extended suitably over $Z$, and the formula for index $D$ still held [4].

The details of how the boundary condition for $D$ connects to the topology over $Z$ are quite delicate and very beautiful. They depend in fact on a new elementary proof of Bott periodicity which is explained in [3]. The starting point here is that loops in $\operatorname{GL}(N, \mathbb{C})$ should be approximated by finite Fourier series

$$
f(Z)=\sum_{-k}^{k} A_{n} Z^{n}
$$

where the coefficients $A_{n}$ are $N \times N$ complex matrices.

All this shows that there is a deep connection between $K$-theory and the index theory of elliptic operators. Much of this has now been absorbed in the more general theories developed by Alain Connes and others.

In looking back over the past, one can sometimes find problems that have been overlooked and might be re-examined. One such problem is to extend the elementary proof of periodicity to the orthogonal group. Here Fourier series, which are appropriate for the circle, have to be replaced by the representation theory of the orthogonal group and spherical harmonics. I am sure Raoul would have liked to see such a programme developed. It would involve explicit formulae of the type he loved.

\section{The heat equation}

One approach to the index theorem which has become very popular, partic-

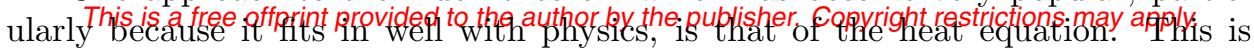
restricted to the Dirac type operators associated to Riemannian geometry, but in 
that context it has significant advantages. Essentially it has a local form and this is required for the APS boundary conditions studied in [11] and the $\eta$-invariant.

The basic analytic idea is extremely simple. Since the self-adjoint operators $D D^{*}$ and $D^{*} D$ have non-negative discrete spectra we can write

$$
\operatorname{Trace}\left(\mathrm{e}^{t D^{*} D}\right)=\sum_{\lambda \geq 0} \mathrm{e}^{t \lambda}
$$

where $\lambda$ runs over the discrete spectrum (with finite multiplicity). It is easy to see that the terms for $\lambda \neq 0$ are the same for $D^{*} D$ and $D D^{*}$, while the difference for $\lambda=0$ gives index $D$. Thus we have the formula

$$
\text { index } D=\operatorname{Trace}\left(\mathrm{e}^{t D^{*} D}\right)-\operatorname{Trace}\left(\mathrm{e}^{t D D^{*}}\right) \text {. }
$$

This is an identity for all $t>0$, but for $t \rightarrow 0$ there are explicit asymptotic expansions for the kernel of the heat operators $\mathrm{e}^{t D^{*} D}$ and $\mathrm{e}^{t D D^{*}}$. In particular (2) gives an explicit integral formula for index $D$.

Unfortunately the asymptotic expansion of the heat kernel is extremely complicated involving high derivatives - how high depends on the dimension of the manifold. However, quite miraculously, the difference of the heat kernel traces in (2) involves remarkable cancellations, all the higher derivatives drop out, and one is left with a much simpler formula which can then be identified as an algebraic expression in the curvature. This represents a certain characteristic class and so we end up with a simple cohomological formula for index $D$.

This algebraic reduction process was discovered first by Patodi [20] and subsequently generalized by Gilkey [16]. These methods appeared complicated to Raoul and me, so together with Patodi we finally [9] explained the whole process in terms of invariant theory. This involved both the classical results of Weyl on invariants of the orthogonal group and the results of Riemannian geometry, reducing everything to the Riemann tensor and the Bianchi identities.

Patodi's original proof involved some slick algebra which Raoul and I could not fathom. I believe this has now been understood by physicists using supersymmetry.

There are two questions arising from all this which I would like to pose.

(1) What is the relation between the supersymmetry proof and the invariant theory proof?

(2) Can the invariant proof, plus the Bianchi identities, be understood as invariant theory of $\operatorname{Diff}(X)$ ? This is, I believe, related to a question raised by Gelfand many years ago.

\section{Fixed-point theorems}

At the Woods Hole conference of 1964 Raoul and I defined a generalization of the index theorem for a map $f: X \rightarrow X$ commuting with an elliptic operator $D$. This involves the Lefschetz number

$$
L(f, D)=\operatorname{Trace}(f \mid \operatorname{ker} D)-\operatorname{Trace}(f \mid \operatorname{coker} D)
$$

Motivated by a problem of Shimura we proposed that, when $f$ has isolated (and transverse) fixed points, this analytic Lefschetz number should be given by a formula of the type

$$
\text { This is a free offprint provided to the author by the publisher. Copyright restrictions may apply. }
$$




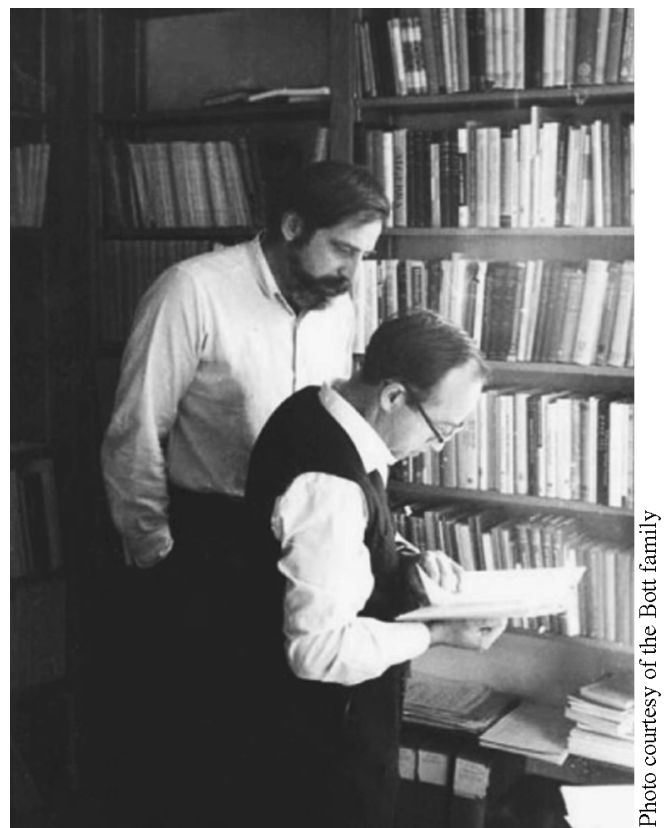

Figure 3. Bott and the author

the sum being over the fixed points $P$ of $f$ and $\nu_{P}(f, D)$ being given by an explicit local formula at $P$.

Having formulated our conjecture, we asked the assembled experts at Woods Hole to compute some simple examples as a check on it. Their verdict was that our conjectured formula was wrong. Fortunately we did not believe them; our formula looked too beautiful! We were in fact justified and our conjecture was eventually proved [5].

This is my version of the events at Woods Hole. Interestingly, none of the experts I consulted could remember the story. As Freud understood, we tend to forget our mistakes and remember our successes!

This fixed-point formula was one of Raoul's favourites. He liked the elegance of the result and its breadth of applications. On the one hand, as we quickly discovered, it implies the famous Hermann Weyl formula for the characters of the irreducible representations of compact semi-simple Lie groups. In a quite different direction it implies the truth of an old conjecture of P. A. Smith for actions of cyclic groups on spheres.

Another surprising consequence of the fixed point theorem was a result discovered by Hirzebruch and myself [10] which showed that, if a spin manifold admits a nontrivial circle action, then the index of the Dirac operator (the $\hat{A}$ genus) is zero (actually we needed an extension of the fixed-point theorem dealing also with higher-dimensional fixed points).

The idea of the proof was simple. One used the explicit formula for the fixedpoint contributions of $L(z, D)$ where $|z|=1$ gives the circle action. Analytically

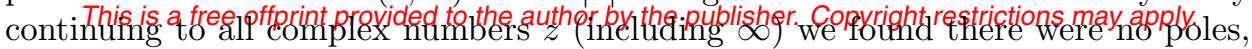
so $L(z, D)$ was independent of $z$ (and actually zero). 
Many years later Witten applied the fixed-point theorem formally in infinite dimensions. He discovered [22] generalizations of my theorem with Hirzebruch. For a whole sequence of representations $R_{n}$ of the orthogonal group the index of the Dirac operator, coupled to $R_{n}$, was rigid, i.e., independent of the circle action.

A rigorous mathematical proof of Witten's result was subsequently obtained by Bott and Taubes [15]. This was modelled on the proof that Hirzebruch and I had given but using modular forms rather than just rational functions. This is closely related to later work of M. Hopkins on elliptic cohomology, and may point to further links between physics and number theory in the future.

\section{Yang-Mills and algebraic curves}

Whenever Raoul and I met we would exchange ideas and frequently a new collaboration would emerge. This happened in Oxford one time when Raoul was returning from a trip to the Tata Institute in Bombay. He was much taken with the study of vector bundles on algebraic curves, the first subject I had studied for my Ph.D. At this time, Yang-Mills theory was flavour of the month for 4-dimensional geometry. Walking across the park one day, on the way to lunch in my college, we began to consider what would come out of Yang-Mills in two dimensions, i.e., for Riemann surfaces or algebraic curves. At first sight we thought the theory would be trivial, but in fact it turned out to be unexpectedly fruitful and resulted in our longest joint paper [6].

The key idea, quite natural to Raoul, was to study the Yang-Mills functional $\left\|F_{A}\right\|^{2}$ on the infinite-dimensional space $\mathcal{A}$ of $G$-connections on a compact Riemann surface $X$.

From the physicists we had learnt of the infinite-dimensional group

$$
\mathcal{G}=\operatorname{Map}(X, G)
$$

of gauge transformations. This acted on $\mathcal{A}$ preserving the Yang-Mills functional, so naively speaking one should pass to the quotient. However Raoul knew from experience that, because the actions were not free, it was better to work with $\mathcal{G}$-equivariant cohomology.

We also discovered the simple but beautiful fact that $\mathcal{A}$ has a natural symplectic structure and that the action of $\mathcal{G}$ defines a moment map

$$
\mu: \mathcal{A} \rightarrow \operatorname{Lie}(\mathcal{G})^{*}
$$

which turns out to be just the curvature $A \rightarrow F_{A}$.

These two facts led us to a new and more direct way of computing the Betti numbers of moduli spaces of flat $G$-bundles over $X$ (or equivalently of moduli spaces of holomorphic $G^{\mathbb{C}}$-bundles). These Betti numbers had been calculated earlier by Harder and Narasimhan [17] by totally different methods. They had worked over finite fields, counting rational points and used the Weil conjectures (subsequently established by Deligne).

Although these two methods are quite different the actual structures of the proofs have remarkable similarities. In both cases one starts with an infinitedimensional situation and reduces by automorphisms. It is a very intriguing question to search for an underlying connection. Two ideas suggest themselves:

(1) Is there an infinite-dimensional version of the Weil conjectures?

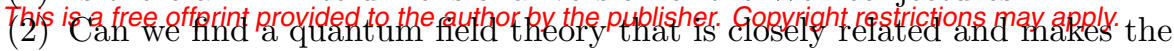
Feynman integral analogous to the Tamagawa measure? 
These questions have been around for over twenty years. Perhaps the time is now right to re-examine them?

Let me end this section with a number of notes.

(3) The method used in [6] turns out to work in general for finite-dimensional geometric invariant theory. This was shown by F. Kirwan [19].

(4) For genus zero (i.e., when $X=S^{2}$ ) [6] is close to the work of Bott on $\Omega G$.

(5) The ideas in [6] have proved useful in physics [23].

(6) In a subsequent paper [7] Raoul and I pursued further the relation between the moment map and equivariant cohomology.

\section{Lacunas}

Although much of my joint work with Raoul centred round elliptic PDE there was one major work on hyperbolic PDE. This was in tripartite collaboration with Lars Gårding who initiated the project and instructed us in the relevant analysis. We were essentially a contract team brought in by Lars because of our expertise in topology and algebraic geometry.

The problem we were brought in to study was that of lacunas for hyperbolic PDE, the generalization of the famous Huygens principle for the propagation of light. Igor Petrowsky [21] had made a detailed study of the problem and established many key results, but the topological techniques of the time were inadequate and the geometrical arguments difficult to understand. Our role was to modernize Petrowsky's presentation making it more intelligible and easier to generalize.

The basic idea is that the fundamental solution of a hyperbolic linear PDE (with constant coefficients) is given, through Fourier transforms, by an explicit integral over a cycle on the complexified characteristic hypersurface. This "period" integral depends on the region of space where we compute the fundamental solution. If it turns out to be zero (as it does trivially outside the entire "light-cone") then the region is a lacuna. There are indeed nontrivial lacunas and determining them is a subtle problem.

Fortunately, once Raoul and I had understood the problem, the modern techniques of algebraic geometry gave us the solution [8]. A key ingredient was a result attributed to Grothendieck which asserted that the cohomology of an affine algebraic variety was given by the complex of rational differential forms. In fact (though I did not realize it at the time) this result is actually implicit in the early paper [18] I wrote with Hodge.

Not only did these modern methods clear up the problems in Petrowsky's work but, under the guidance of Lars Gårding, they led to substantial generalizations.

\section{References}

1. M. F. Atiyah, Bott periodicity and the index of elliptic operators, Quart. J. Math. Oxford Ser. (2) 19 (1968), 113-140.

2. _ Raoul Harry Bott, Biographical Memoirs of Fellows — Royal Society 53 (2007), 63-76.

3. M. F. Atiyah and R. Bott, On the periodicity theorem for complex vector bundles, Acta Math. 112 (1964), 229-247.

4. The index problem for manifolds with boundary, Differential Analysis, Bombay Colloq., 1964, Oxford Univ. Press, London, 1964, pp. 175-186.

5. _ A Lefschetz fixed point formula for elliptic differential operators, Bull. Amer. Math. Sofnis 7 is (1966) off 45 -250 250 vided to the author by the publisher. Copyright restrictions may apply.

6. ㄴ. The Yang-Mills equations over Riemann surfaces, Philos. Trans. Roy. Soc. London Ser. A 308 (1983), no. 1505, 523-615. 


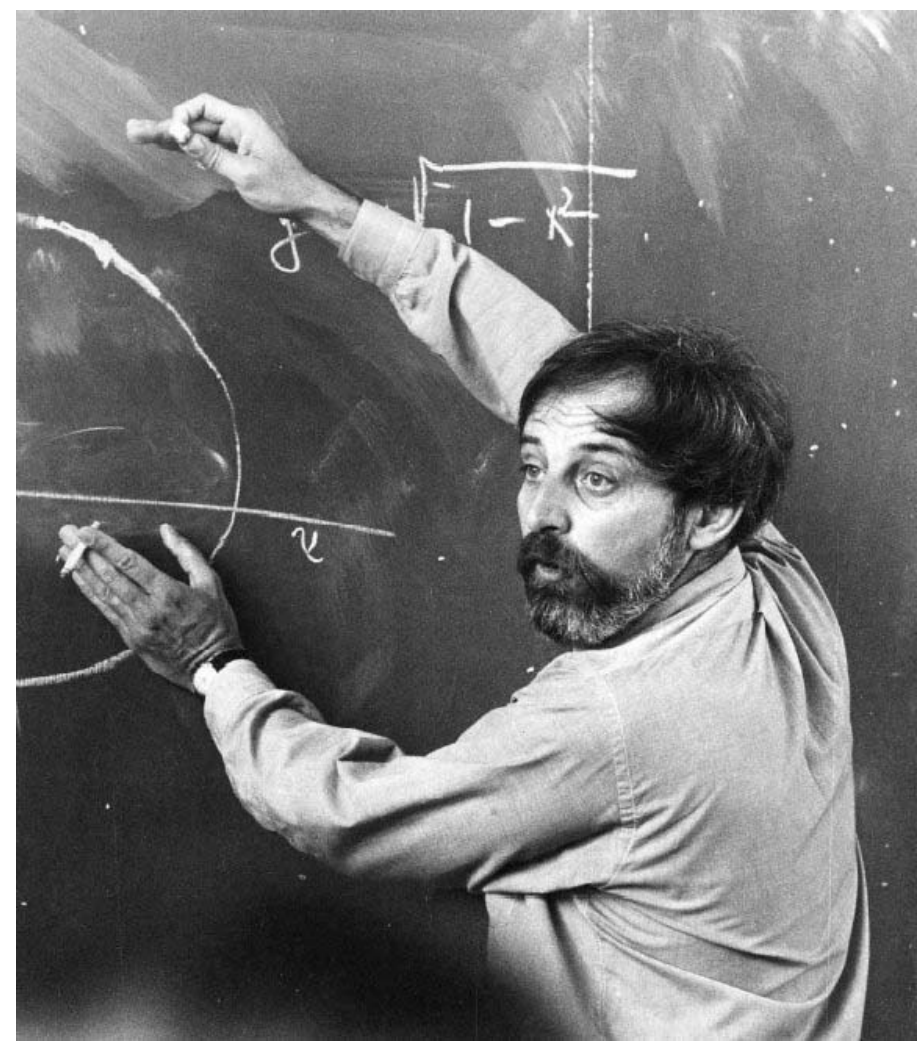

Figure 4. Raoul Bott lecturing at the Universität Bonn. (C)Wolfgang Vollrath

7. The moment map and equivariant cohomology, Topology 23 (1984), no. 1, 1-28.

8. M. F. Atiyah, R. Bott, and L. Gårding, Lacunas for hyperbolic differential operators with constant coefficients. I, Acta Math. 124 (1970), 109-189.

9. M. F. Atiyah, R. Bott, and V. K. Patodi, On the heat equation and the index theorem, Invent. Math. 19 (1973), 279-330.

10. M. F. Atiyah and F. Hirzebruch, Spin-manifolds and group actions, Essays on Topology and Related Topics (Mémoires dédiés à Georges de Rham), Springer, New York, 1970, pp. 18-28.

11. M. F. Atiyah, V. K. Patodi, and I. M. Singer, Spectral asymmetry and Riemannian geometry. I, Math. Proc. Cambridge Philos. Soc. 77 (1975), 43-69.

12. M. F. Atiyah and I. M. Singer, The index of elliptic operators on compact manifolds, Bull. Amer. Math. Soc. 69 (1963), 422-433.

13. R. Bott, The stable homotopy of the classical groups, Ann. of Math. (2) 70 (1959), 313-337. 14. Quelques remarques sur les théorèmes de périodicité, Bull. Soc. Math. France $\mathbf{8 7}$ (1959), 293-310.

15. R. Bott and C. Taubes, On the rigidity theorems of Witten, J. Amer. Math. Soc. 2 (1989), no. $1,137-186$.

16. P. B. Gilkey, Curvature and the eigenvalues of the Laplacian for elliptic complexes, Advances in Math. 10 (1973), 344-382.

17. G. Harder and M. S. Narasimhan, On the cohomology groups of moduli spaces of vector

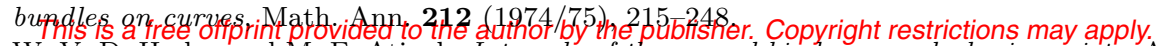

18. W. V. D. Hodge and M. F. Atiyah, Integrals of the second kind on an algebraic variety, Ann. of Math. (2) 62 (1955), 56-91. 
19. F. C. Kirwan, Cohomology of quotients in symplectic and algebraic geometry, Math. Notes, vol. 31, Princeton Univ. Press, Princeton, NJ, 1984.

20. V. K. Patodi, An analytic proof of Riemann-Roch-Hirzebruch theorem for Kähler manifolds, J. Differential Geometry 5 (1971), 251-283.

21. I. Petrowsky, On the diffusion of waves and the lacunas for hyperbolic equations, Rec. Math. [Mat. Sbornik] N. S. 17(59) (1945), 289-370.

22. E. Witten, Elliptic genera and quantum field theory, Comm. Math. Phys. 109 (1987), no. 4, 525-536.

23. _ Two-dimensional gauge theories revisited, J. Geom. Phys. 9 (1992), no. 4, 303-368.

The School of Mathematics, The University of Edinburgh, James Clerk Maxwell Building, The King's Buildings, Mayfield Road, Edinburgh, EH9 3JZ, UK

E-mail address: m.atiyah@ed.ac.uk 